DAMTP

$\mathrm{M} / \mathrm{C}$ TH $98 / 24$

\title{
PERTURBATIVE EVOLUTION AND REGGE BEHAVIOUR
}

\author{
J R Cudell \\ Institut de Physique, Université de Liège \\ A Donnachie \\ Department of Physics, Manchester University \\ P V Landshoff \\ DAMTP, Cambridge University*
}

\begin{abstract}
The known analytic properties of the Compton amplitude at small $Q^{2}$ place significant constraints on its behaviour at large $Q^{2}$. This calls for a re-evaluation of the role of perturbative evolution in past fits to data.
\end{abstract}

\section{Introduction}

There is a widespread belief that the rise at small $x$ observed at HERA in the proton structure function is associated with the presence of a singularity at $N=0$ in the Mellin transform of the DGLAP splitting function. Indeed, fits to structure functions typically incorporate perturbative QCD evolution in which this singularity plays a key role ${ }^{[1]}$, but ignore an old ${ }^{[2]}$ but very basic piece of knowledge: that at small $x$ a structure function is governed by Regge theory ${ }^{[3]}$. Recently, two of us have made ${ }^{[4]}$ an extremely successful fit to all small- $x$ data, from $Q^{2}=0$ up to $2000 \mathrm{GeV}^{2}$, in which we concentrate on the Regge theory and ignore the requirements imposed by perturbative evolution. It is the purpose of this paper to try to bring the two together. In particular, we argue that, by analytically continuing in $Q^{2}$, one can conclude that the singularities in the complex $N$-plane of the Mellin transform of the structure function must already be present at small $Q^{2}$, and the perturbative evolution cannot generate new singularities that appear only at high $Q^{2}$. In particular, the role customarily assigned to the $N=0$ singularity is not sustainable.

Regge theory has its basis in the analyticity properties of the appropriate scattering amplitude, in this case the virtual Compton amplitude whose imaginary part at $t=0$ contains the familar structure functions $F_{1}\left(x, Q^{2}\right)$ and $F_{2}\left(x, Q^{2}\right)$. A rigorous analysis of analyticity properties of scattering amplitudes is extremely difficult ${ }^{[5]}$. So instead one assumes ${ }^{[6]}$ that the region of analyticity is the same as the analyticity region shared by all the infinite number of Feynman graphs that one can draw for the amplitude, ignoring the fact that in a strong-coupling régime the numerical values of these graphs are irrelevant because the perturbation series diverges. From this approach one may deduce that, both for the real-photon Compton amplitude and for $Q^{2}<0$, there is sufficient analyticity to derive the Regge theory - if anything, more so when $Q^{2}<0$. The analyticity properties are determined by the masses of the physical hadrons, and it is crucial that none of these has zero mass. The fact that gluons perhaps have zero mass is irrelevant because they are not physical particles, even though often we pretend they are in calculations.

* email addresses: cudell@gw.unipc.ulg.ac.be, ad@a3.ph.man.ac.uk, pvl@damtp.cam.ac.uk 
With no zero-mass particle exchange, there is a finite "Lehmann ellipse" in the $t$-channel, within which the $t$-channel partial wave series converges for $t>4 m^{2}$, where $m$ is the proton mass, and for suitable values of $s-$ or, equivalently, $\nu$ if we are considering deep inelastic scattering. For example, for the amplitude $T_{1}\left(\nu, t, Q^{2}\right)$ whose imaginary part at $t=0$ is $F_{1}\left(x, Q^{2}\right)$ with $x=Q^{2} / 2 \nu$,

$$
T_{1}\left(\nu, t, Q^{2}\right)=\sum_{\ell=0}^{\infty}(2 \ell+1) a_{\ell}\left(t, Q^{2}\right) P_{\ell}\left(\cos \theta_{t}\right)
$$

where $\nu$ must be such that

$$
\cos \theta_{t}=-\frac{\nu-\frac{1}{4} t}{\sqrt{\left(\frac{1}{4} t+Q^{2}\right)\left(\frac{1}{4} t-m^{2}\right)}}
$$

has absolute magnitude no greater than 1 . The Froissart-Gribov technique ${ }^{[3]}$ then uses a fixed- $t$ dispersion relation to define an analytic function $a\left(\ell, t, Q^{2}\right)$ which is equal to $a_{\ell}\left(t, Q^{2}\right)$ when the angular momentum $\ell$ takes the values $0,1,2,3, \ldots$, but is defined also for complex values of $\ell$ and has suitable behaviour when $|\ell| \rightarrow \infty$ to allow a Watson-Sommerfeld transform of the partial-wave series (1.1a). This expresses the series as an integral*:

$$
T_{1}\left(\nu, t, Q^{2}\right)=\frac{1}{2 i} \int_{C} \frac{(2 \ell+1) P_{\ell}\left(-\cos \theta_{t}\right)}{\sin \pi \ell} a\left(\ell, t, Q^{2}\right)
$$

with $P_{\ell}\left(\cos \theta_{t}\right)$ the analytic continuation of the Legendre polynomial to complex $\ell$. The contour $C$ is initially that of figure 1a, wrapped arround the positive real- $\ell$ axis on which are located the zeros of the denominator $\sin (\pi \ell)$. But the properties of $a\left(\ell, t, Q^{2}\right)$ allow it to be distorted to become parallel to the imaginary- $\ell$ axis, as in figure $1 \mathrm{~b}$, so extending the range of values of $\nu$ and $t$ for which the representation of $T_{1}\left(\nu, t, Q^{2}\right)$ is valid.

From what is known about the analyticity structure, it is rather certain that the positions of the singularities of $a\left(\ell, t, Q^{2}\right)$ in the complex $\ell$ plane do not depend on $Q^{2}$, only on $t$. If we analytically continue in $t$ to the value we need, $t=0$, a singularity may try to cross the contour $C$, and we must distort the contour again so as to avoid this happening. Depending on whether the singularity is a branch point or a pole, we then have either figure $2 \mathrm{a}$ or $2 \mathrm{~b}$. In the case of a pole

$$
a\left(\ell, t, Q^{2}\right) \sim \frac{\beta\left(Q^{2}, t\right)}{\ell-\alpha(t)}
$$

the integration around it yields a contribution

$$
\frac{\pi \beta\left(Q^{2}, t\right) P_{\alpha(t)}\left(\cos \theta_{t}\right)}{\sin \pi \alpha(t)}
$$

to $T_{1}\left(\nu, t, Q^{2}\right)$. Now make an analytic continuation so that $\nu$ becomes large,

$$
\nu \gg Q m
$$

* More correctly, the Froissart-Gribov technique splits the summation (1.1a) into two, one over even $\ell$ and the other over odd $\ell$. Each is converted into an integral, with an "even-signature" amplitude $a^{+}\left(\ell, t, Q^{2}\right)$ that equals $a_{\ell}\left(t, Q^{2}\right)$ for $\ell=0,2,4, \ldots$ and an "odd-signature amplitude $a^{-}\left(\ell, t, Q^{2}\right)$ that equals $a_{\ell}\left(t, Q^{2}\right)$ for $\ell=1,3,5, \ldots$. 

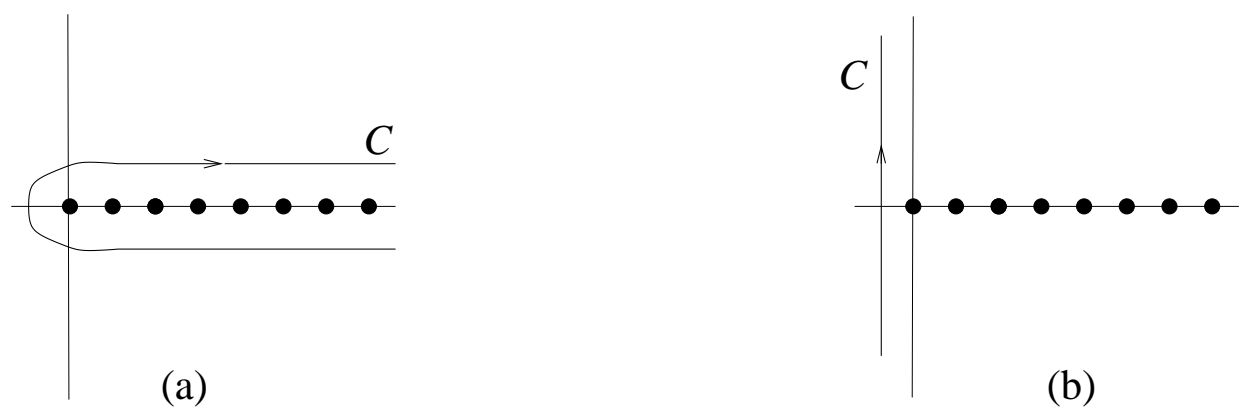

Figure 1: Contour $C$ in the complex $\ell$ plane for the integral (1.2)

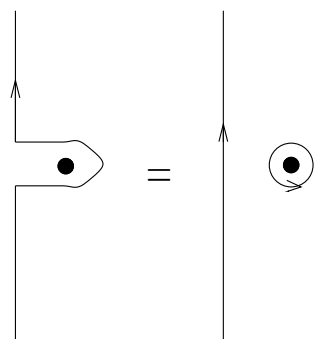

(a)

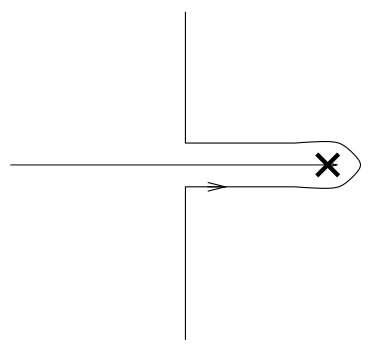

(b)

Figure 2: Distortion of the contour $C$ caused by (a) a pole, or (b) a branch point of $a\left(\ell, t, Q^{2}\right)$ crossing it.

Then $\left|\cos \theta_{t}\right| \gg 1$. For large $z$,

$$
P_{\ell}(z) \sim z^{\ell}
$$

so the Watson-Sommerfeld transform (1.2) becomes essentially a Mellin transform[ ${ }^{[6]}$ and the "Regge pole" $\alpha(t)$ contributes at $t=0$

$$
b_{1}\left(Q^{2}\right) \nu^{\alpha(0)}
$$

to the large- $\nu$ behaviour of $T_{1}\left(\nu, 0, Q^{2}\right)$. Here, $b_{1}\left(Q^{2}\right)$ is a constant multiple of $\beta\left(Q^{2}, 0\right)$. In the case of $T_{2}\left(\nu, 0, Q^{2}\right)$ its definition includes a kinematic factor which reduces the power of $\nu$ by one unit, so since $\nu=Q^{2} / x$ this gives

$$
F_{2}\left(x, Q^{2}\right) \sim f\left(Q^{2}\right) x^{1-\alpha(0)}
$$

Regge theory gives no information about the function $f\left(Q^{2}\right)$, other than that they are analytic functions with singularities whose locations are known ${ }^{[6]}$. The power $(1-\alpha(0))$ is independent of $Q^{2}$.

In the case where the singularity that crosses the contour $C$ is a branch point at $\ell=\alpha_{c}(t)$, dragging a branch cut with it as shown in figure $2 \mathrm{~b}$, the simple power of $x$ in (1.5) is replaced with

$$
F_{2}\left(x, Q^{2}\right) \sim \int^{\alpha_{c}(0)} d \ell f\left(\ell, Q^{2}\right) x^{1-\ell}
$$

which results in $F_{2}\left(x, Q^{2}\right) \sim x^{1-\alpha_{c}(0)}$ times an unknown function of $Q^{2}$ and $\log (1 / x)$. Again, the power $\left(1-\alpha_{c}(0)\right)$ is independent of $Q^{2}$. One knows, from unitarity ${ }^{[3]}$, that if there are poles in the complex $\ell$ plane, there must also be branch points. On the principle that it is usually the best strategy to try the simplest possible assumption first, and because there is good evidence that it is correct in 

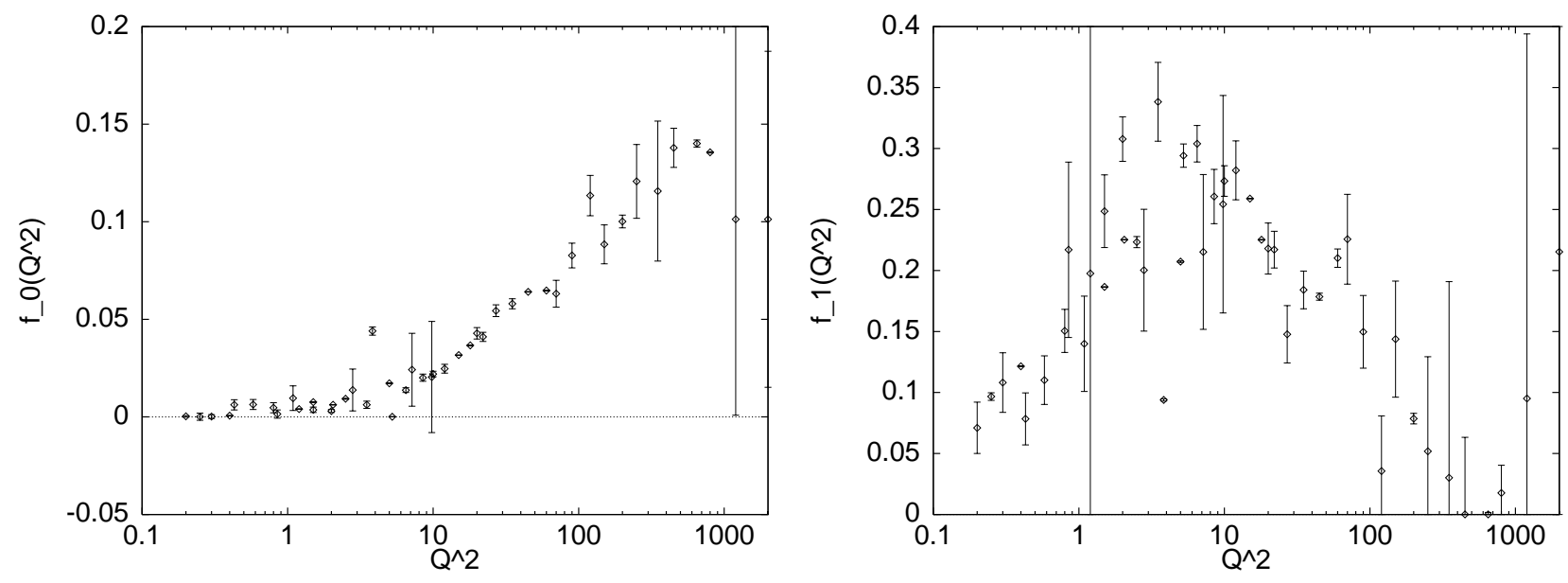

Figure 3: The coefficient functions $f_{0}$ and $f_{1}$ extracted from data at each $Q^{2}$; the error bars are from MINUIT

purely hadronic interactions ${ }^{[7]}$, in reference 4 we tested the hypothesis that the contribution to the structure function $F_{2}$ at small $x$ from branch points is much weaker than from poles. We made a fit of the form

$$
F_{2}\left(x, Q^{2}\right) \sim \sum_{i=0}^{2} f_{i}\left(Q^{2}\right) x^{-\epsilon_{i}}
$$

We fixed the values of two of the powers from our knowledge ${ }^{[7]}$ of hadron-hadron total cross-sections:

$$
\begin{array}{cc}
\epsilon_{1}=0.08 \quad \text { ("soft pomeron" exchange) } \\
\epsilon_{2}=-0.45 \quad(\rho, \omega, f, a \text { exchange })
\end{array}
$$

and extracted the value of $\epsilon_{0}$ from the small- $x$ data - we used all data for which $x<0.07$ and $Q^{2}$ ranged from 0 to $2000 \mathrm{GeV}^{2}$. This gave $\epsilon_{0} \approx 0.4$ and provided an excellent fit to the data over the whole range of $Q^{2}$.

In our fit, we constrained each of the three coefficent functions $f_{i}\left(Q^{2}\right)$ to vanish as $\left(Q^{2}\right)^{1+\epsilon_{i}}$ as $Q^{2} \rightarrow 0$. Gauge invariance requires the total $F_{2}$ to vanish linearly with $Q^{2}$ at fixed $\nu$, and these constraints ensure that each of the three separate terms in (1.6a) have this property. From the data, we found that the "hard-pomeron" term is present already at small $Q^{2}$, though its coefficient function is very small, but it grows approximately logarithmically for large $Q^{2}$. The soft-pomeron coefficient function, on the other hand, rises quickly with $Q^{2}$ for small $Q^{2}$, peaks at a value of $Q^{2}$ between 5 and $10 \mathrm{GeV}^{2}$, and then becomes small at large $Q^{2}$. See figure 3. The data do not impose much constraint on how the meson-exchange coefficent function behaves for large $Q^{2}$.

Regge theory gives no quantitative information about the coefficient functions $f_{i}\left(Q^{2}\right)$, but at large $Q^{2}$ they should evolve according to perturbative QCD. That is, provided they correspond to leadingtwist contributions, their behaviour at large $Q^{2}$ should be governed by the DGLAP equation ${ }^{[1]}$. The constraint from Regge theory is that the positions of singularities in the complex $\ell$ plane should should not change with $Q^{2}$, and that new singularities do not suddenly appear as $Q^{2}$ increases. In our fit to the data ${ }^{[4]}$, we have shown that this is fully supported by experiment. It is our purpose in this paper to discuss it further. 


\section{The DGLAP equation: general theory}

We write the singlet DGLAP equation as ${ }^{[8]}$

$$
\frac{\partial}{\partial \log Q^{2}} \mathbf{u}\left(x, Q^{2}\right)=\int_{x}^{1} d z \boldsymbol{\pi}\left(z, \alpha_{S}\left(Q^{2}\right)\right) \mathbf{u}\left(\frac{x}{z}, Q^{2}\right)
$$

where

$$
\mathbf{u}=\left(\begin{array}{c}
x \sum_{f}\left(q_{f}+\bar{q}_{f}\right) \\
x G
\end{array}\right)
$$

Introduce the Mellin transform

$$
\mathbf{U}\left(N, Q^{2}\right)=\int_{0}^{1} d x x^{N-1} \mathbf{u}\left(x, Q^{2}\right)
$$

so that

$$
\mathbf{u}\left(x, Q^{2}\right)=\int \frac{d N}{2 \pi i} x^{-N} \mathbf{U}\left(N, Q^{2}\right)
$$

As we have explained, $\mathbf{U}\left(N, Q^{2}\right)$ is essentially the complex-partial-wave amplitude $\mathbf{a}\left(\ell, t, Q^{2}\right)$ at $t=0$, with $\ell=N+1$, so that a power contribution

$$
f\left(Q^{2}\right) x^{-\epsilon}
$$

to $F_{2}\left(x, Q^{2}\right)$ corresponds to a pole

$$
\frac{\mathbf{f}\left(Q^{2}\right)}{N-\epsilon}
$$

in $\mathbf{U}\left(N, Q^{2}\right)$. If the small- $x$ behaviour of $F_{2}$ is (1.6a), the rightmost singularity of $\mathbf{U}\left(N, Q^{2}\right)$ in the complex $N$-plane is a pole at $N=\epsilon_{0}$, and the integration in (2.2b) initially should be along a line

$$
\operatorname{Re} N=N_{0} \quad \text { with } N_{0}>\epsilon_{0}
$$

It is evident from its definition (2.2a) that $\mathbf{U}\left(N, Q^{2}\right)$ is analytic to the right of this line in the complex $N$-plane, and for $\operatorname{Re} N \rightarrow+\infty$ it goes to 0 for physical values of $x$, that is $0<x<1$. In what follows we shall analytically continue $\mathbf{U}\left(N, Q^{2}\right)$ to values of $N$ such that $\operatorname{Re} N<\epsilon_{0}$; then it is no longer given by the representation $(2.2 \mathrm{a})$, which then diverges.

Insert the Mellin-transform representation (2.2b) into the DGLAP equation (2.1a):

$$
\int \frac{d N}{2 \pi i} x^{-N} \frac{\partial}{\partial \log Q^{2}} \mathbf{U}\left(N, Q^{2}\right)=\int \frac{d N}{2 \pi i} x^{-N} \boldsymbol{\Pi}\left(N, x, \alpha_{S}\left(Q^{2}\right)\right) \mathbf{U}\left(N, Q^{2}\right)
$$

where

$$
\boldsymbol{\Pi}\left(N, x, \alpha_{S}\left(Q^{2}\right)\right)=\int_{x}^{1} d z z^{N} \boldsymbol{\pi}\left(z, \alpha_{S}\left(Q^{2}\right)\right)
$$

If the rightmost singularity of $\mathbf{U}\left(N, Q^{2}\right)$ in the complex $N$-plane is a pole at $N=\epsilon_{0}$, with residue $\mathbf{f}_{0}\left(Q^{2}\right)$, we may move the contour of $N$ integration to the left past this pole and so pick up a contribution to each side of (2.5a) that behaves as $x^{-\epsilon_{0}}$. This gives the differential equation

$$
\frac{\partial}{\partial \log Q^{2}} \mathbf{f}_{0}\left(Q^{2}\right)=\overline{\mathbf{\Pi}}\left(\epsilon_{0}, \alpha_{S}\left(Q^{2}\right)\right) \mathbf{f}_{0}\left(Q^{2}\right)
$$


with $\bar{\Pi}\left(\epsilon_{0}, \alpha_{S}\left(Q^{2}\right)\right)$ the analytic continuation to $N=\epsilon_{0}$ of

$$
\overline{\mathbf{\Pi}}\left(N, \alpha_{S}\left(Q^{2}\right)\right)=\int_{0}^{1} d z z^{N} \boldsymbol{\pi}\left(z, \alpha_{S}\left(Q^{2}\right)\right)
$$

To leading order in $\alpha_{S}\left(Q^{2}\right)$, the difference between $\bar{\Pi}\left(\epsilon_{0}, \alpha_{S}\left(Q^{2}\right)\right)$ and $\boldsymbol{\Pi}\left(\epsilon_{0}, x, \alpha_{S}\left(Q^{2}\right)\right)$ behaves as $x^{\epsilon_{0}}$ at $x=0$ and therefore is negligibly small.

A differential equation such as (2.6) actually applies to the coefficient function of each power term $\mathbf{f}\left(Q^{2}\right) x^{-\epsilon}$ in $\mathbf{u}\left(x, Q^{2}\right)$, not just the leading one. That is, a term that behaves as any power at some value of $Q^{2}$ remains a power. However, there are apparent complications, arising from the fact that, to lowest order in $\alpha_{S}\left(Q^{2}\right), \Pi\left(N, x, \alpha_{S}\left(Q^{2}\right)\right)$ is singular at $N=0$. We address this matter in the next section.

In lowest order, the splitting function $\pi\left(z, \alpha_{S}\left(Q^{2}\right)\right)$ introduced in (2.1a) is simply proportional to $\alpha_{S}\left(Q^{2}\right)$, and likewise its Mellin transform $\bar{\Pi}\left(N, \alpha_{S}\left(Q^{2}\right)\right)$ of $(2.6 \mathrm{~b})$, so that (2.6a) reads

$$
\tau \frac{\partial}{\partial \tau} \mathbf{f}_{0}\left(Q^{2}\right)=\mathbf{B}\left(\epsilon_{0}\right) \mathbf{f}_{0}\left(Q^{2}\right) \quad \tau=\log \frac{Q^{2}}{\Lambda^{2}}
$$

The solution to $(2.7)$ is

$$
\mathbf{f}_{0}\left(Q^{2}\right)=\exp \left(T\left(Q^{2}\right) \mathbf{B}\left(\epsilon_{0}\right)\right) \mathbf{f}_{0}\left(Q_{0}^{2}\right) \quad T\left(Q^{2}\right)=\log \frac{\log Q^{2} / \Lambda^{2}}{\log Q_{0}^{2} / \Lambda^{2}}
$$

In terms of the standard anomalous-dimension matrix $\gamma(j)$,

$$
\mathbf{B}(N)=C\left(\begin{array}{cc}
\gamma_{q q}(N-1) & 2 N_{f} \gamma_{q G}(N-1) \\
\gamma_{G q}(N-1) & \gamma_{G G}(N-1)
\end{array}\right) \quad C=\frac{6}{33-2 N_{f}}
$$

The elements of $\gamma(j-1)$ are plotted in figure 4.9 of reference 8 whose authors have kindly provided us with the output of their computations ${ }^{*}$, from which in figure 4 we plot the elements of the matrix $\mathbf{B}(N)$ in the case $N_{f}=4$, and in figure 5 its eigenvalues $\lambda_{ \pm}(N)$.

If we expand $\mathbf{f}_{0}\left(Q^{2}\right)$ as a linear combination of the two eigenvectors of $\mathbf{B}\left(\epsilon_{0}\right)$ :

$$
\mathbf{f}_{0}\left(Q^{2}\right)=\phi_{0+}\left(Q^{2}\right) \mathbf{v}_{+}\left(\epsilon_{0}\right)+\phi_{0-}\left(Q^{2}\right) \mathbf{v}_{-}\left(\epsilon_{0}\right)
$$

where

$$
\begin{gathered}
\mathbf{v}_{ \pm}(N)=K_{ \pm}(N)\left(\begin{array}{c}
B_{q G}(N) \\
\lambda_{ \pm}(N)-B_{q q}(N)
\end{array}\right) \\
\left(K_{ \pm}(N)\right)^{-2}=\left(B_{q G}(N)\right)^{2}+\left(\lambda_{ \pm}(N)-B_{q q}(N)\right)^{2}
\end{gathered}
$$

then

$$
\phi_{0 \pm}\left(Q^{2}\right)=\left(\frac{\log Q^{2} / \Lambda^{2}}{\log Q_{0}^{2} / \Lambda^{2}}\right)^{\lambda_{ \pm}\left(\epsilon_{0}\right)} \phi_{0 \pm}\left(Q_{0}^{2}\right)
$$

According to figure 5, one eigenvalue $\lambda_{-}$is negative, so for large $Q^{2}$ only the first term on the righthand-side of (2.10a) survives, and then the hard-pomeron coefficient functions satisfy

$$
\frac{f_{0 q}\left(Q^{2}\right)}{f_{0 G}\left(Q^{2}\right)}=\frac{B_{q G}\left(\epsilon_{0}\right)}{\lambda_{+}\left(\epsilon_{0}\right)-B_{q q}\left(\epsilon_{0}\right)}
$$

\footnotetext{
* It is not made clear in the book that the $q g$ plot includes the necessary factor $2 N_{f}$.
} 

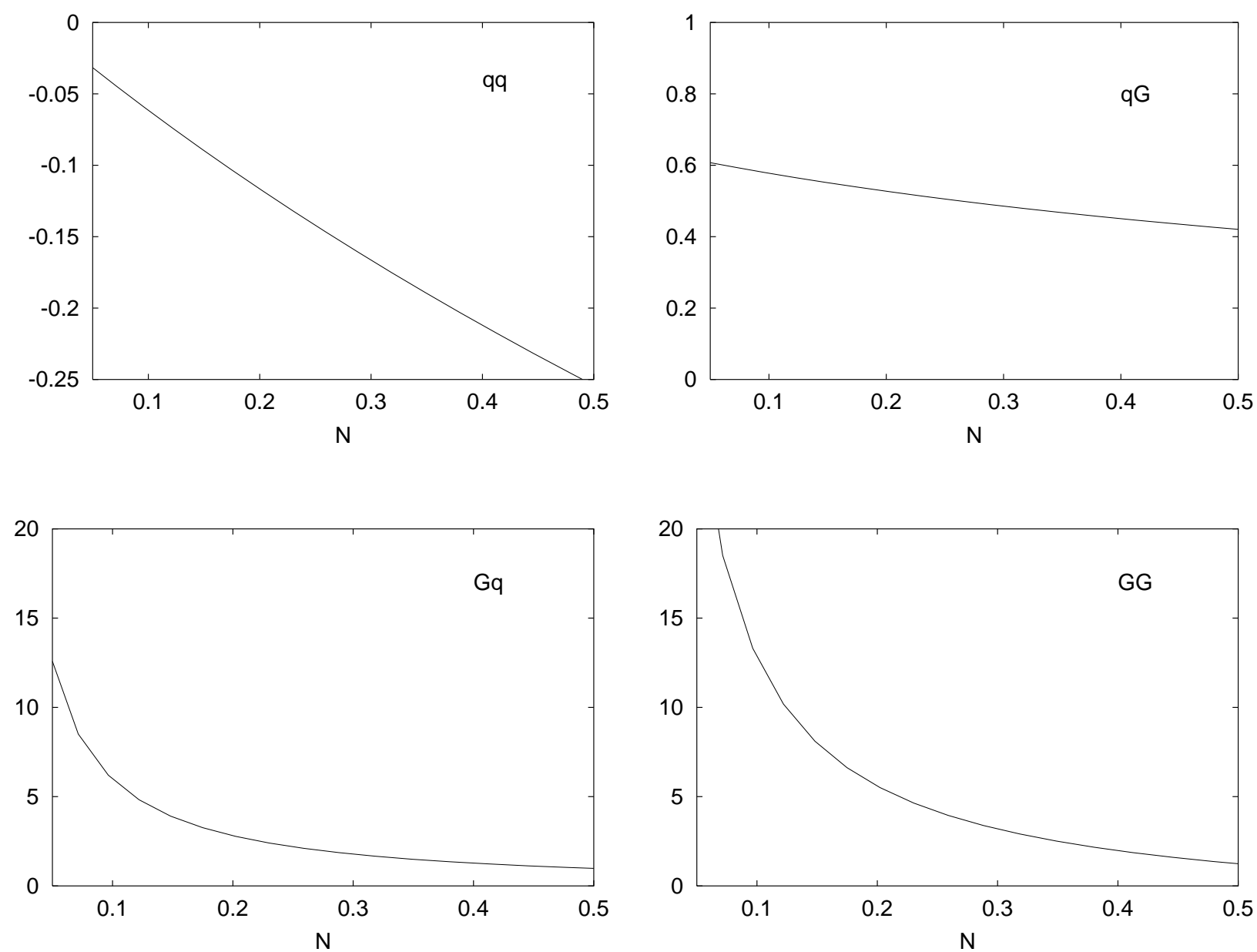

Figure 4: Elements of the matrix $\mathbf{B}(N)$

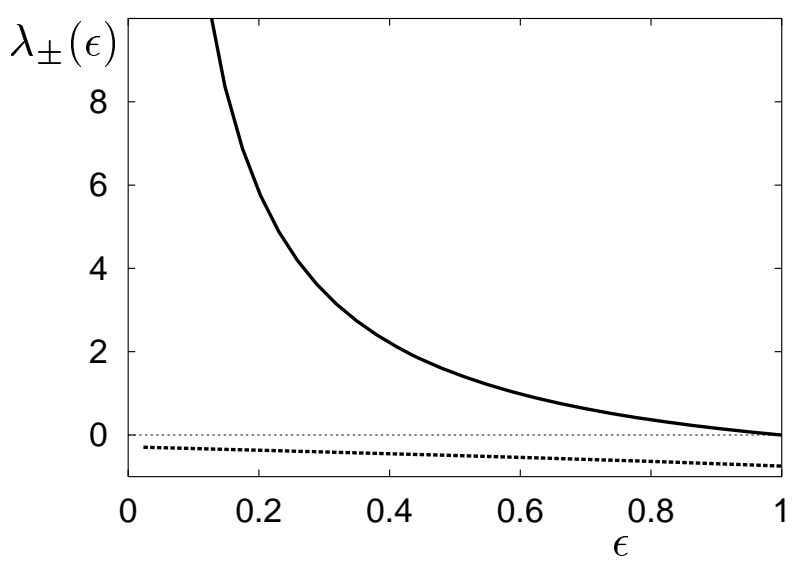

Figure 5: Eigenvalues $\lambda_{ \pm}(N)$ of the matrix $\mathbf{B}(N)$ 
when $Q^{2}$ is large enough.

In our previous fit to the structure-function data we used the forms

$$
\begin{gathered}
f_{0}\left(Q^{2}\right)=A_{0}\left(\frac{Q^{2}}{Q^{2}+a_{0}}\right)^{1+\epsilon_{0}}\left(1+X \log \left(1+\frac{Q^{2}}{Q_{0}^{2}}\right)\right) \\
f_{1}\left(Q^{2}\right)=A_{1}\left(\frac{Q^{2}}{Q^{2}+a_{1}}\right)^{1+\epsilon_{1}} \frac{1}{1+\sqrt{Q^{2} / Q_{1}^{2}}} \\
f_{2}\left(Q^{2}\right)=A_{2}\left(\frac{Q^{2}}{Q^{2}+a_{2}}\right)^{1+\epsilon_{2}}
\end{gathered}
$$

where the soft-pomeron power $\epsilon_{1}$ was constrained to be 0.08 and the meson-exchange power $\epsilon_{2}$ was fixed at -0.45 . This resulted in a $\chi^{2}$ just less than 1.02 per data point for the 539 data points for which $x<0.07$, including the real-photon total cross-section measurements for which $\sqrt{ } s>6 \mathrm{GeV}$. The fit gave the "hard-pomeron" power $\epsilon_{0}$ to be about 0.4 for which, according to figure $5 \mathrm{a}, \lambda_{+}\left(\epsilon_{0}\right)$ is close to 2. On the other hand, figure 5 a would give $\lambda_{+}\left(\epsilon_{1}\right)$ somewhat larger, which is in obvious conflict with the behaviour of $f_{1}\left(Q^{2}\right)$ at large $Q^{2}$ extracted from the data: see figure $3 \mathrm{~b}$. This reinforces our view that the soft-pomeron term is higher twist. In a new fit, we have therefore left its form unchanged, and replaced the hard-pomeron coefficent function with

$$
f_{0}\left(Q^{2}\right)=A_{0}\left(\frac{Q^{2}}{Q^{2}+a_{0}}\right)^{1+\epsilon_{0}}\left(1+X \log \left(1+\frac{Q^{2}}{\Lambda^{2}}\right)^{\lambda_{+}\left(\epsilon_{0}\right)}\right)
$$

so there is one fewer parameter. In principle, if the meson-exchange term is leading twist (which presumably it is) we should also include a logarithmic factor in its high- $Q^{2}$ behaviour, raised to power $\lambda_{+}\left(\epsilon_{2}\right)$ obtained by analytically continuing $\lambda_{+}(N)$ to negative $N$. However, this term gives only a very small contribution to the high- $Q^{2}$ data points and so we have left it as in $(2.12 \mathrm{a})$. We find that making the change $(2.12 \mathrm{~b})$ increases the $\chi^{2}$ very slightly, to a little over 1.05 per data point.

Our fit omits any contribution from a singularity at $N=0$. We now explain why this is justified.

\section{Singularity at $N=0$}

To lowest-order in $\alpha_{S}$ the splitting function $\pi\left(z, \alpha_{S}\left(Q^{2}\right)\right)$ has a $z^{-1}$ singularity at $z=0$, so that its Mellin transform $\overline{\mathbf{\Pi}}\left(N, \alpha_{S}\left(Q^{2}\right)\right)$, defined in (2.5b), has an $N^{-1}$ singularity at $N=0$. This means that every power $x^{-\epsilon}$, whether $\epsilon$ is whether positive or negative, generates through (2.5a) an additional singularity in $\mathbf{U}\left(N, Q^{2}\right)$ at $N=0$ as $Q^{2}$ increases. The evolution of this $N=0$ singularity is not controlled just by the small- $x$ behaviour of the structure function.

To investigate these matters, write the lowest-order splitting function as

$$
\pi\left(z, \alpha_{S}\left(Q^{2}\right)\right)=\frac{\alpha_{S}\left(Q^{2}\right)}{2 \pi}\left[\hat{\mathbf{p}}(z)+\mathbf{p}_{0} z^{-1}\right]
$$

where $\hat{\mathbf{p}}(z)$ is regular at $z=0$. Inserting this into $(2.5)$ gives

$$
\int \frac{d N}{2 \pi i} x^{-N} \frac{\partial}{\partial \log Q^{2}} \mathbf{U}\left(N, Q^{2}\right)=\frac{\alpha_{S}\left(Q^{2}\right)}{2 \pi} \int \frac{d N}{2 \pi i} x^{-N}\left\{\hat{\mathbf{P}}(N, x)+N^{-1} \mathbf{p}_{0}\left(1-x^{N}\right)\right\} \mathbf{U}\left(N, Q^{2}\right)
$$


where $\hat{\mathbf{P}}(N, x)$ is a Mellin transform of $\hat{\mathbf{p}}(z)$ like $(2.5 \mathrm{~b})$. It vanishes so rapidly at $x=0$ that it is a good approximation to replace it with $\hat{\mathbf{P}}(N, 0)$. Because $\mathbf{U}\left(N, Q^{2}\right)$ is analytic to the right of the contour of the $N$ integration and, from its definition (2.2a), vanishes when $|N| \rightarrow \infty$, the last term inside the curly bracket integrates to zero, and the inverse Mellin transform of (3.2) is just

$$
\begin{aligned}
\frac{\partial}{\partial \log Q^{2}} \mathbf{U}\left(N, Q^{2}\right) & \left.\sim \alpha_{S}\left(Q^{2}\right)[\hat{\mathbf{P}}(N, 0))+N^{-1} \mathbf{p}_{0}\right] \mathbf{U}\left(N, Q^{2}\right) \\
& =\frac{\alpha_{S}\left(Q^{2}\right)}{2 \pi} \mathbf{P}(N) \mathbf{U}\left(N, Q^{2}\right)
\end{aligned}
$$

Evidently a pole $(2.3 \mathrm{~b})$ at $N=\epsilon$ in $\mathbf{U}\left(N, Q^{2}\right)$ gives a pole in $\left(\partial / \partial \log Q^{2}\right) \mathbf{U}\left(N, Q^{2}\right)$. By taking the residue at the pole of both sides of (3.3) we see that the coefficient function $\mathbf{f}\left(Q^{2}\right)$ satisfies a differential equation like (2.6a), involving the analytic continuation in $N$ of $\overline{\mathbf{P}}(N)$ to $N=\epsilon$. This is true for either sign of $\epsilon$.

The solution to $(3.3)$ is

$$
\mathbf{U}\left(N, Q^{2}\right) \sim \exp \left[C \log \frac{\log \left(Q^{2} / \Lambda^{2}\right)}{\log \left(Q_{0}^{2} / \Lambda^{2}\right)} \mathbf{P}(N)\right] \mathbf{U}\left(N, Q_{0}^{2}\right)
$$

where the constant $C$ is defined in (2.9). It is evident that, to use this equation, we must make some assumption about $\mathbf{U}\left(N, Q_{0}^{2}\right)$ at some value $Q_{0}^{2}$ of $Q^{2}$. The common assumption ${ }^{[9]}$ is that, for suitable choice of $Q_{0}^{2}$, it is regular at $N=0$. Indeed, there is no reason to suppose that, for $Q_{0}^{2}$ chosen to be 0 or very small, $\mathbf{U}\left(N, Q_{0}^{2}\right)$ is singular at $N=0$. That is, although the real-photon Regge amplitude $a\left(\ell, t, Q^{2}\right)$ has a wrong-signature fixed pole ${ }^{[10]}$ at $\ell=1$, there is no reason to believe that at small $Q^{2}$ it has also a right-signature singularity in the spin-averaged amplitude. Nevertheless, as soon as $Q^{2}$ becomes large enough for the evolution to be correctly described by the DGLAP equation, we learn from (3.4) that $\mathbf{U}\left(N, Q_{0}^{2}\right)$ acquires an essential singularity at $N=0$. A function such as $\mathbf{U}\left(N, Q_{0}^{2}\right)$ that is analytic at $N=0$ for a finite range of $Q^{2}$ cannot suddenly acquire a fixed singularity at $N=0$ when it is analytically continued in $Q^{2}$.

The explanation* of this contradiction is that it is wrong to use the lowest-order approximation to the splitting function, $\Pi\left(N, x, \alpha_{S}\left(Q^{2}\right)\right)$, with its $N^{-1}$ singularity, for any purpose where its behaviour near $N=0$ is important. The higher-order terms in its expansion involve more and more powers of $\alpha_{S} / N$ and near $N=0$ these powers become larger and larger, so that the expansion cannot possibly converge. Our general argument, that new singularities cannot suddenly appear in $\mathbf{U}\left(N, Q^{2}\right)$ as $Q^{2}$ increases, strongly suggests that, if one were to resum the powers of $\alpha_{S} / N$ one would find that in fact $\Pi\left(N, x, \alpha_{S}\left(Q^{2}\right)\right)$ is regular at $N=0$.

This is suppported by what is known about this resummation ${ }^{[11]}$. The DGLAP anomalous dimension $\gamma_{G G}(N-1)$ in $(2.9)$ is equal to the BFKL anomalous dimension $\bar{\gamma}(N)$ calculated to all orders in $\bar{\alpha}_{S} / N$. It is obtained by solving the equation

$$
\chi\left(\frac{\alpha_{S}}{2 \pi} \bar{\gamma}(N)\right)=\frac{\pi N}{3 \alpha_{S}}
$$

where $\chi(x)$ is the Lipatov characteristic function. To lowest order in $\alpha_{S}$

$$
\chi(x)=-2 \gamma_{E}-\psi(x)-\psi(1-x)
$$

\footnotetext{
* We are grateful to Guido Altarelli and Stefano Catani for helping us to understand this
} 


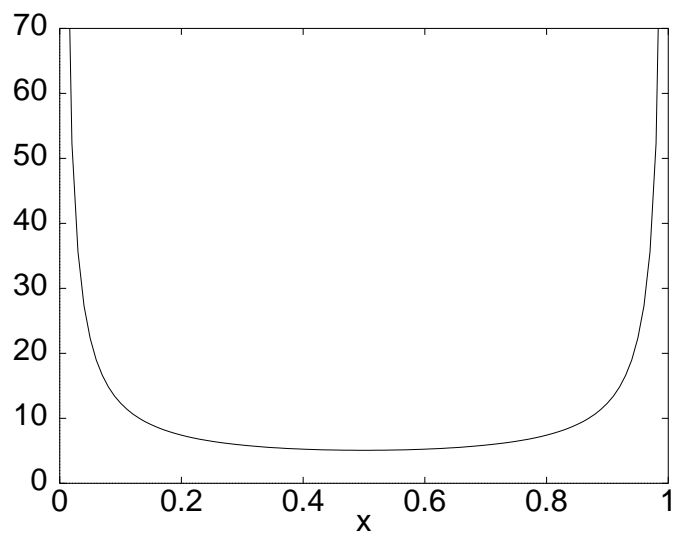

Figure 6: Plot of the lowest-order approximation (3.5b) to the Lipatov characteristic function

which is plotted in figure 6. From this plot, one sees that there are two complex-conjugate solutions for $\bar{\gamma}(N)$ at $N=0$; these are finite. If one expands the solution to the implicit equations (3.5) for $\bar{\gamma}(N)$ in powers of $\alpha_{S}$, the terms in the expansion are singular at $N=0$ because the digamma function $\psi(\gamma)$ is singular at $\gamma=0$, but this expansion is completely invalid near $N=0$.

We now know ${ }^{[12]}$ that the lowest-order approximation $(3.5 \mathrm{~b})$ to the Lipatov characteristic function is inadequate. When the next term in the expansion is included the curve in figure 6 is pulled downwards and $\bar{\gamma}(N)$ is no longer complex at $N=0$, but it is still nonsingular, as our general argument has demanded. However, because the second term in the expansion of the Lipatov characteristic function is so large, it is likely that subsequent terms are too, and so we do not yet have a reliable estimate of just how large $\bar{\gamma}(N)$ is at $N=0$. But we know that it is nonsingular.

Therefore, that in fitting data one should not include in the splitting function any singularity at $N=0$. The only $N$-plane singularities are those already present in the structure function at small $Q^{2}$. These are the standard singularities of Regge theory - the soft pomeron, the mesons, and possibly also a hard pomeron. There may well be branch-point singularities, but the data are consistent with the assumption that their contribution is relatively small at presently accessible values of $x$ and $Q^{2}$. This is the approach we followed in our previous paper ${ }^{[4]}$, and we found that it was highly successful. We found also that the usual attitude to higher twists is more than a little suspect: rather than making a fit that starts by assuming they are as small as the data will allow, one should recognise that the data strongly suggest that a significant part of the structure function at $Q^{2}$ values of $5 \mathrm{GeV}^{2}$ or less is higher twist - the soft-pomeron term, and maybe also meson exchange.

This research is supported in part by the EU Programme "Training and Mobility of Researchers", Networks "Hadronic Physics with High Energy Electromagnetic Probes" (contract FMRX-CT96-0008) and "Quantum Chromodynamics and the Deep Structure of Elementary Particles" (contract FMRX-CT98-0194), and by PPARC

\section{References}

1 V.N. Gribov and L.N. Lipatov, Sov. J. Nucl. Phys. 15 (1972) 438 and 675

L.N. Lipatov, Sov. J. Nucl. Phys. 20 (1975) 94

Yu. L. Dokshitser, Sov. J. JETP 46 (1977) 641

G. Altarelli and G. Parisi, Nucl. Phys. B126 (1977) 298 
2 P V Landshoff, J C Polkinghorne and R D Short, Nuclear Physics B28 (1970) 210

3 P D B Collins, Introduction to Regge Theory and High Energy Physics, Cambridge University Press (1977)

4 A Donnachie and P V Landshoff, Physics Letters B437 (1998) 408

5 R F Streater and A S Wightman, PCT, Spin 6 Statistics, and All That, W A Benjamin (1964)

6 R J Eden, P V Landshoff, D I Olive and J C Polkinghorne, The Analytic S-Matrix, Cambridge University Press (1966)

7 A Donnachie and P V Landshoff, Physics Letters B296 (1992) 227

8 R K Ellis, W J Stirling and B R Webber, QCD and Collider Physics Cambridge University Press (1996)

9 A De Rujula, S L Glashow, H D Politzer, S B Treiman and A Zee, Physical Review D10 (1974) 1649

10 P V Landshoff and J C Polkinghorne, Physical Review D5 (1972) 2056

11 J Forshaw and D A Ross, Quantum chromodynamics and the pomeron, Cambridge University Press (1997)

12 V S Fadin and L N Lipatov, Physics Letters B429 (1998) 127

G Camici and M Ciafaloni, Physics Letters B430 (1998) 349 